\title{
El dilema del consenso por superposición rawlsiano
}

\author{
JOAN VERGÉS GIFRA \\ Universidad de Girona
}

RESUMEN. En este trabajo presentamos una objeción al liberalismo político de John Rawls. La objeción puede formularse en forma de un dilema que afecta especialmente a la noción de consenso por superposición: o bien el consenso por superposición no ofrece ninguna razón categórica o justificativa a favor de los dos principios de justicia rawlsianos y, por tanto, es irrelevante, o bien tal noción presupone la aceptación de determinados caracteres o conceptos propios de un liberalismo comprehensivo $y$, por consiguiente, la concepción de la justicia como equidad no es tan política como Rawls sugiere.

\section{Introducción}

Según Rawls, el argumento completo a favor de su concepción de la justicia consta de dos fases '. La primera fase, que podemos llamar argumento contractualista, consiste en presentar la concepción de la justicia como equidad como la concepción política -esto es, freestanding con respecto a las doctrinas comprehensivas razonables de la sociedad civil- resultante de un argumento contractualista sofisticado. La segunda fase, que podemos llamar ar-
ABSTRACT. In this paper we present an objection against the idea of overlapping consensus, nuclear to John Rawls' political liberalism. The objection can be put in a dilemmatic form: either an overlapping consensus plays no significant role when it comes to justify the two principles of the conception of justice as fairness, or such idea presupposes the acceptation of some traits or concepts particular to a comprehensive liberalism. 
te, el problema que aquí queremos tratar se hace patente tan pronto como formulamos preguntas del tipo: si la justicia como equidad es realmente una concepción política, y ser una concepción política significa estar justificada con independencia de las doctrinas comprehensivas de la sociedad, ¿por qué tenemos que creer que los ciudadanos han de poder ver una congruencia entre ella y su propia concepción del bien? Si el argumento contractualista es correcto, ¿para qué se precisa - a efectos justificativos, categóricos-conjeturar que la concepción de la justicia resultante generará efectivamente un consenso por superposición a su alrededor?

\section{Suficiencia de la primera fase o sospecha de un punto de vista comprehensivo}

Pero ¿cómo podemos responder a estas preguntas? Propongo hacer el siguiente razonamiento. Concedamos, en primer lugar, que la defensa completa de los dos principios de justicia requiere superar las dos fases de argumentación. Imaginemos que acabamos de terminar la primera $y$ que el resultado ha sido la elección de los dos principios de justicia de la concepción rawlsiana. Nos hallamos en la segunda fase. ¿Qué puede suceder en esta segunda fase? Obviamente pueden pasar tres cosas. Es posible que todas las doctrinas comprehensivas que haya en la sociedad estén de acuerdo con los dos principios de justicia. Es posible que ninguna los suscriba. $O$ bien puede suceder que algunas doctrinas comprehensivas no estén de acuerdo con los dos principios de justicia, pero otras sí.

La primera y segunda posibilidades son demasiado inverosímiles. Ciertamente: se hace difícil pensar que todas las doctrinas comprehensivas de una sociedad radicalmente plural coincidirán alguna vez o bien en defender, o bien en rechazar al unísono unos y los mismos principios de ordenamiento social -principios a los cuales cabe atribuir cierta fuerza, puesto que han sido escogidos en la primera fase-. La gracia del problema del pluralismo es que suele haber discrepancia de posturas. Por lo tanto, lo más sensato es pensar que unas doctrinas defenderán una cosa, otras doctrinas defenderán otra y, en consecuencia, tendremos que diferenciar las buenas de las malas respuestas - las posturas tolerantes de las intolerantes, por ejemplo-. Por consiguiente, tenemos que trabajar con la tercera posibilidad: en cuanto llegamos al final de la primera fase, unas doctrinas darán su apoyo a los principios de justicia seleccionados y otras no. Ahora bien, como es obvio, en este punto tan sólo nos será útil saber qué dicen al respecto las doctrinas que sean razonables; porque tan sólo lo que digan ellas - y no lo que digan las doctrinas irrazonables - podrá modificar nuestra opinión sobre la idoneidad moral de los principios resultantes del argumento contractualista. En definitiva, pues, la gran pregunta es saber si una doctrina comprehensiva razonable chocará alguna vez o no con los dos principios de justicia.

De acuerdo con Brian Barry, el liberalismo político de Rawls oscila entre afirmar que las doctrinas comprehensivas razonables no chocarán nunca con los dos principios de justicia y afirmar que podrían estar en conflicto ${ }^{2}$. De entrada, parece que una doctrina comprehensiva razonable en ningún caso podrá contradecir los dos principios de justicia, puesto que será razonable en virtud de la aceptación de estos dos principios. Es decir, el criterio de razonabilidad aplicado a las doctrinas comprehensivas será la aceptación de los dos principios de justicia. En otras palabras: una doctrina será razonable si pudiera ser una de las concepciones del bien que las partes en la posición original podrían imaginar que sus representados podrían tener. Si una doctrina no es razonable el poder político podrá obligar a sus miembros a atenerse a los principios de justicia, a pesar de que no los acepten. 
Este uso coercitivo del poder político no es ilegítimo, en absoluto. Según Barry, tal es el criterio de razonabilidad que Rawls aplicó a las concepciones del bien en $A$ Theory of Justice, y es el criterio que a veces parece adoptar en Political Liberalism $^{3}$. Ahora bien, fijémonos en que este criterio hace irrelevante la segunda fase de la argumentación. La irrelevancia del argumento consensualista se hace patente en el hecho de que parece formar parte de una estrategia viciosamente circular. El círculo sigue más o menos el siguiente dibujo: «¿Cómo se pueden poner a prueba los dos principios de justicia?», pregunta el intérprete a Rawls. «Pues demostrando que pueden ganarse el apoyo de las doctrinas comprehensivas razonables», responde él. «Pero ¿cómo sabemos que una doctrina es razonable?», insiste el intérprete. «Es razonable si acepta los dos principios de justicia; en caso contrario, no lo es», responde Rawls» ${ }^{4}$.

Así pues, a fin de poder creer que la segunda fase de la argumentación es realmente relevante es preciso negar antes que el criterio de razonabilidad que ha de aplicarse a las doctrinas comprehensivas es la aceptación de los dos principios de justicia. Tan sólo entonces tendrá sentido que nos planteemos la cuestión de si estos dos principios conseguirán atraerse el apoyo de las doctrinas comprehensivas razonables en un consenso por superposición. Es decir, la cuestión del apoyo de las doctrinas comprehensivas razonables tiene que estar abierta al final de la primera fase. Tenemos que creer que no se ha dicho todo aún. Si no sabemos si los dos principios de justicia serán aceptados por todas las doctrinas comprehensivas razonables, entonces podemos creer que en el caso de que alguna de ellas muestre reticencias, tal vez necesitemos retocarlos.

Ahora bien, esta posibilidad comporta consecuencias graves para el liberalismo político. Porque, como se ve, presupone que existe un sentido pre-político de «razonable» cuando decimos que una doctrina comprehensiva es razonable. El problema, por consiguiente, ahora será explicar las razones que tenemos para juzgar que unas doctrinas son razonables mientras que otras no, y ello sin poder apelar a ninguna concepción política de la justicia. Es en este punto que uno empieza a sospechar que, al final, los comunitaristas tal vez tengan razón y la concepción de la justicia de Rawls presupone, cuando no toda una concepción particular de la vida buena, sí al menos algunas nociones controvertidas que afectarán inevitablemente la percepción que se puede tener de la vida buena. Es decir, surge la sospecha de que Rawls juega con las cartas marcadas y que tan sólo considera «razonables» aquellas doctrinas comprehensivas que respetan unas determinadas características controvertidas sobre lo que debemos entender por vida buena. La acusación es ligeramente diferente de la que hemos formulado antes. Antes hemos dicho que el argumento del consenso por superposición era circular; ahora decimos que el argumento presupone que las doctrinas comprehensivas que podrán formar parte de él serán de un tipo controvertido comprehensivamente. Por lo tanto, la concepción de la justicia como equidad no es tan política como Rawls nos quiere hacer creer.

La sospecha de los comunitaristas, como decimos, es que detrás de la concepción de la justicia como equidad se esconde una concepción de la vida buena eminentemente liberal que mucha gente no estaría dispuesta a aceptar. Barry y Kymlicka también tienen la misma sospecha. En términos rawlsianos: no creen que la justicia como equidad sea una concepción independiente - freestanding - . Sin embargo, ni Barry ni Kymlicka lamentan esta circunstancia. Al contrario, se alegran de ello. Pues, tal como dice Barry, la «perspectiva liberal», es decir, la adopción de determinadas actitudes liberales controvertidas, no tan sólo es condición suficien- 
te para poder aceptar las instituciones liberales, sino también es condición necesaria. Por lo tanto, si Rawls quiere defender correctamente los dos principios de justicia deberá apelar expresamente a valores exclusivamente liberales ${ }^{5}$. De hecho, aunque no quiere admitirlo, ya lo hace.

\section{Escepticismo comprehensivo}

¿Qué valores liberales parece presuponer Rawls que las doctrinas comprehensivas deberán suscribir para poder ser consideradas razonables? A fin de responder esta pregunta, Barry nos invita a fijarnos en el requisito de neutralidad que pretende satisfacer la concepción política de la justicia y nos detengamos a pensar cómo lo podrán cumplir aquellas personas que, por ejemplo, profesen una religión como la católica. Para empezar, la concepción de la justicia como equidad presupone que estas personas tendrán que haber aprendido a realizar una clara distinción entre aquellos actos o creencias incorrectos que son objeto de legítima prohibición pública porque perjudican a uno o más ciudadanos en cuanto ciudadanos; y aquellos actos o creencias, también incorrectos, que no son objeto de legítima prohibición porque no perjudican a ningún ciudadano -en cuanto ciudadano, se entiende- ${ }^{6}$. Ahora bien, para poder realizar tal distinción deberán creer que los actos y creencias del primer grupo son incorrectos en un sentido más fuerte que los del segundo. En realidad, tendrán que aceptar que las creencias del segundo grupo conciernen más bien la opinión personal que pueda tener cada persona - es decir, que es normal que haya gente que piense de otro modo y que tal cosa no es ninguna desgracia--. Sin embargo,

[tratar la doctrina de la Iglesia] en términos de opinión personal es profundamente conflictivo con las afirmaciones de la propia Iglesia sobre su propia doctrina. Se puede decir exactamente lo mismo de aquellos que derivan las nociones de correcto e incorrecto directamente de la autoridad de un libro como la Biblia, en vez de la autoridad de una organización como la Iglesia Católica Romana. En cualquier caso, para que alguien esté dispuesto a decir «la homosexualidad es mala, pero esto tan sólo es mi opinión personal» tiene que haberse tragado ya una buena dosis de liberalismo ?

En cada doctrina comprehensiva podemos distinguir dos partes, según Barry ${ }^{8}$. Por un lado, está el contenido de las creencias que la caracterizan. En este sentido, cada doctrina tiene las creencias que tiene: el catolicismo, por ejemplo, mantiene actualmente, que la homosexualidad es pecado. Pero aparte del contenido doctrinal, cada doctrina comprehensiva también posee una actitud o creencia de segundo orden sobre el estatus epistemológico de sus creencias de primer orden o contenido doctrinal. Ahora bien, hay dos tipos de actitudes o creencias de segundo orden sobre el estatus epistemológico de las creencias de primer orden. Está la actitud del «dogmático», según la cual nadie que sea razonable no puede rechazar su concepción de primer orden $-\mathrm{y}$ en este sentido un católico no aceptará que alguien crea que la homosexualidad es pecado-. Y está la actitud del «escéptico», según la cual la otra gente puede rechazar razonablemente la concepción de primer orden que uno tiene - y en este sentido un católico tolerará que alguien crea que la homosexualidad no es pecado - La actitud escéptica es lo que explica, pues, que un católico pueda tener el segundo sentido de incorrecto al que acabamos de referirnos. No sólo eso: según Barry, el escepticismo es la base fundamental del liberalismo. $Y$ también debería ser la base de la concepción de la justicia de Rawls.

Pero Rawls afirma que su concepción de la justicia no se puede basar en una teoría tan controvertida como el escepticismo. En caso de hacerlo, muchas doctrinas comprehensivas no la aceptarían ${ }^{9}$. Ahora bien, ello ocurrirá así, en cualquier caso, 
porque en cada doctrina comprehensiva hay efectivamente el componente de segundo orden del que habla Barry. Por lo tanto, la pregunta que tenemos que formular a Rawls es si no cree que una doctrina comprehensiva, para ser razonable, ha de ser «escéptica» y no «dogmática», tal como Barry define estos términos. En realidad, en algunos sitios Rawls realiza afirmaciones muy parecidas a la caracterización del escepticismo de Barry, como cuando sugiere que, en el fondo, lo que hace que una doctrina comprehensiva sea razonable no es el contenido de lo que defiende sino cómo lo defiende ${ }^{10}$. Según el liberalismo político, recordemoslo, una persona es razonable si acepta la tesis de las cargas del juicio. Sin embargo, muchos intérpretes opinan que aceptar las cargas del juicio supone ir más allá de constatar la dificultad que experimentamos los humanos para ponernos de acuerdo en determinadas cuestiones ${ }^{11}$. Teniendo en cuenta las consecuencias que extrae de tal tesis, supone bastante más. Barry, cuando menos, cree que equivale a aceptar el escepticismo, tal como él lo entiende. Sea como fuere, lo que parece claro es que muchas doctrinas comprehensivas no podrán tener una actitud de segundo orden como la que requiere la concepción política de la justicia rawlsiana; a menos, claro, que ya se hayan convertido al liberalismo, en un grado eminentemente comprehensivo ${ }^{12}$. Pero entonces la segunda fase del argumento rawlsiano - y la noción de consenso por superposición, con su correspondiente noción de tolerancia- perderá casi todo su interés.

\section{Autonomía comprehensiva}

Will Kymlicka también se suma al frente que ha abierto Barry contra el liberalismo político ${ }^{13}$. Pero en vez de fijarse directamente en la actitud de segundo orden que uno deberá tener con respecto a sus creencias de primer orden, él se fija en la noción de autonomía que aparentemente subyace a la exigencia de adoptar tal actitud ${ }^{14}$. Es decir, aborda directamente el valor moral que la sostiene: el valor de la autonomía. Como es sabido, la concepción de la persona de Rawls presupone que las personas tienen un interés prioritario (highest-order interest) en vivir un vida buena que puedan considerar propia; esto implica que tienen un interés prioritario en examinar, revisar y modificar, si cabe, la concepción del bien que en cualquier momento puedan tener. Ahora bien, esta noción de autonomía ha sido muy cuestionada por los comunitaristas. Porque el comunitarismo afirma que las personas no pueden separarse, así como así, de los fines, de los valores, de los vínculos que están incardinados (embedded) en la posición que ocupan en la comunidad. Pues estos fines, valores y vínculos son constitutivos de su personalidad moral. Ante esto, como también es sabido, Rawls ha replicado que la noción de autonomía de su concepción de la justicia tan sólo es válida a nivel político; a nivel comprehensivo, uno puede rechazarla. Es decir, tan sólo se aplica a las personas consideradas como ciudadanos. Gracias a esto, uno puede trazar una distinción clara entre aquellas demandas que pueden llegar a requerir hasta la prohibición legítima de determinados actos o creencias, y aquellas otras demandas que no son más que la expresión de opiniones personales y, por consiguiente, no justifican la coerción de la discrepancia. Pues bien, Kymlicka plantea la cuestión de si tal uso parcial de la noción de autonomía es coherente. Dicho de otra modo: se pregunta si puede haber personas que rechacen la noción a nivel personal o comprehensivo, pero crean que deben aceptarla a nivel político.

En este punto, Kymlicka recuerda la respuesta que ha dado Rawls al problema. Rawls cree que las personas que no tienen una doctrina comprehensiva liberal -puesto que suscriben una doctrina religiosa, 
por ejemplo- estarán dispuestas a aceptar la autonomía política porque de este modo se asegurarán el ejercicio futuro libre de sus propias convicciones. Es decir, no verán ningún coste en el hecho de que en la sociedad donde viven los ciudadanos tengan libertad de conciencia y creencia. En cuanto se percaten de que las sociedades modernas son esencialmente plurales verán que el reconocimiento de las libertades básicas de los individuos es la única forma de acomodar la pluralidad de doctrinas comprehensivas. Pero es aquí donde Rawls se equivoca, opina Kymlicka. O mejor dicho, es aquí donde Rawls demuestra que su concepción política presupone la aceptación de una perspectiva liberal particular controvertida. Porque no es nada claro que los miembros de doctrinas comprehensivas religiosas no vean ningún coste en esta solución política. $Y$ no es nada claro que no consideren este coste muy superior a la adopción de otra solución al problema del pluralismo:

[Pero] las minorías no-liberales verán un coste en la aceptación de la concepción política de la persona de Rawls; a saber, que excluye cualquier sistema de derechos de grupo que limita el derecho de los individuos a revisar sus concepciones del bien. Por ejemplo, impide que una minoría religiosa prohíba la apostasía y la proselitización o la posibilidad de que sus niños aprendan otras formas de vida ${ }^{\text {is. }}$.

En efecto, existe otra solución que también puede acomodar sin problema el hecho del pluralismo y que desde el punto de vista de las doctrinas comprehensivas religiosas será mucho más tentadora. Se trata de la solución que históricamente representa el Imperio Otomano ${ }^{16}$. El Imperio Otomano no fue una sociedad políticamente liberal; es decir, no reconocía los derechos y las libertades individuales fundamentales de las democracias liberales modernas. Sin embargo, a pesar de basarse en la religión musulmana que profesaba una mayoría de sus súbditos, fue un imperio notablemente tolerante con las minorías religiosas y étnicas. De hecho, durante mucho tiempo reconoció oficialmente el derecho de las comunidades minoritarias -la judía, la griega ortodoxa y la armenia ortodoxa - a autogobernarse y a velar por la integridad y respeto de la doctrina en su propia comunidad. Así pues, no es cierto que, ante el pluralismo de doctrinas comprehensivas, una sociedad esté obligada a adoptar el tipo de instituciones liberales de las democracias modernas. La solución de la garantía constitucional de derechos y libertades individuales constituye tan sólo una respuesta al pluralismo doctrinal; pero no es la única. Kymlicka cree que Rawls no se ha percatado de esta posibilidad - la posibilidad de acomodar el pluralismo recurriendo a la idea de derechos de grupo- $y$, por esta razón, supone que la solución más natural para el pluralismo es la aceptación de un sistema de libertades individuales de tipo democrático liberal.

Pero esto no es el del todo cierto ${ }^{17}$. En realidad, sí se ha dado cuenta. De hecho, en The Law of Peoples Rawls describe una sociedad idealmente «decente»-que él llama Kazanistán - inspirada en el modelo de tolerancia y respeto de la pluralidad religiosa del Imperio Otomano ${ }^{18}$. Según Rawls, las sociedades decentes son miembros de pleno derecho en la Sociedad de Pueblos bien ordenados porque están de acuerdo con los principios del derecho de gentes que también aceptan las sociedades liberales; por consiguiente, merecen un trato equitativo por parte de las sociedades liberales. Una sociedad decente como Kazanistán no es liberal puesto que se fundamenta en una concepción de la persona de tipo hegeliano, dice Rawls; es decir, considera que el bien de sus ciudadanos está vinculada a la pertenencia a determinadas comunidades o grupos religiosos ${ }^{19}$. Casi podríamos decir -con Kymlicka - que Kazanistán, como el Imperio Otomano, es «una especie de 
hipercomunitarismo» ${ }^{20}$. Recordemos, por otro lado, que una sociedad es decente si respeta los derechos humanos de sus miembros, no es agresiva hacia los demás pueblos, su sistema legal impone deberes y obligaciones a todas las personas del territorio y contiene una idea de justicia como bien común en la que creen sinceramente los administradores de justicia y los políticos.

Con todo, el reconocimiento por parte de Rawls de la posibilidad de acomodar el pluralismo mediante el reconocimiento de derechos de grupo no hace sino empeorar todavía más la situación de su teoría. Nos percatamos del peligro que corre cuando, con Kymlicka, le preguntamos cómo piensa defender que el sistema liberal es superior al sistema basado en derechos de grupo - si lo piensa hacer, claro-. En The Law of Peoples Rawls afirma que, a pesar de que los regímenes liberales son razonables y los otros miembros de la Sociedad de Pueblos son meramente decentes, y a pesar de que la decencia es una idea normativa «del mismo tipo que la idea de razonabilidad, pero más débil (cubre menos aspectos que la idea de razonabilidad)» ${ }^{21}$, no podemos decir que los primeros son superiores a los segundos. Hacer tal cosa podría ser ofensivo para las sociedades decentes y equivaldría a no tratarlas equitativamente como iguales ${ }^{22}$. Ahora bien, la respuesta de Rawls no es demasiado convincente. Porque no es nada claro que podamos evitar siempre la pregunta de qué sistema político es mejor moralmente. En el caso de las relaciones internacionales tal vez sea más fácil convencernos de que podemos esquivar el problema, porque al lado de la cuestión de la justicia tenemos las cuestiones de la soberanía y el derecho al autorrespeto de un pueblo - si bien incluso entonces es difícil dar la razón a Rawls - 23. De todos modos, lo que queremos decir es que el problema sobrepasa el marco de las relaciones internacionales y afecta, queramos o no, la cuestión de la justicia en el ámbito doméstico. Y entonces sí que no parece posible que Rawls pueda evitar la cuestión de qué sistema o valores son mejores y por qué. Para percatarnos de ello, tan sólo necesitamos plantearnos la siguiente hipótesis. Imaginemos que una persona que vive en una sociedad rawlsiana posee una «doctrina comprehensiva decente» y cree que la mejor forma de respetar lo que ésta le exige es defender la transformación de la sociedad democrática liberal en una sociedad decente del mismo tipo que Kazanistán. O mejor: imaginemos que un colectivo mayoritario de ciudadanos, todos ellos miembros de una religión determinada, aspira a convertir el país en un país confesional como Kazanistán. Hagamos un esfuerzo adicional: figurémonos que una gran cantidad de estadounidenses quisieran seguir el camino inverso que ha seguido hasta ahora su país y aspirasen a hacer de él un nuevo Kazanistán o un nuevo Imperio Otomano. ¿Rawls no se atrevería a decirles nada? Es difícil creer que no ${ }^{24}$.

Pero ¿qué les podría decir? Se me ocurren tres posibilidades: i) Tal vez Rawls tendrá la tentación de contestarles que la cultura política pública de Estados Unidos - a diferencia de la cultura política de los pueblos decentes que el gobierno estadounidense tolera en el foro internacionales eminentemente liberal $y$, por consiguiente, este tipo de propuestas de reforma no serán consideradas adecuadas para la gente. Pero una respuesta así parece más propia de un político que de un filósofo político. La cuestión que el reformador decente plantea no tiene nada que ver con qué aspecto tiene hoy la cultura política de Estados Unidos o qué creen los estadounidenses, sino qué deberían creer. ii) Entonces Rawls tal vez querrá replicar que el tipo de cambios que el reformador decente propone no serían jamás aceptados en la posición original de la justicia como equidad. Pero ante esta respuesta el reformador decente siempre podría replicar que 
sus reformas constituyen una enmienda a la totalidad a la justicia como equidad y, por consiguiente, no puede aceptar una descripción de una situación de elección ideal como la de Rawls; él también podría ofrecer un argumento contractualista tipo posición original que le favoreciera particularmente -incorporando en ella, por ejemplo, una concepción de la persona comunitarista- iii) La tercera respuesta que Rawls podría ofrecer al reformador decente sería la siguiente: «Vuestra propuesta de reforma está equivocada, porque concibe erróneamente algunos aspectos básicos actualmente de la moralidad y de la persona.»

Tal es la respuesta que Kymlicka y Barry querrían que diera Rawls. Ambos están convencidos de que el liberalismo político rawlsiano se autoengaña si cree que es neutral con las doctrinas comprehensivas no liberales cuando justifica los dos principios de justicia: el análisis muestra que en realidad presupone valores y actitudes propiamente liberales. Más le valdría, pues, que lo reconociera de entrada. Así podría defender mejor, más claramente, su postura normativa de fondo - especialmente sus presupuestos igualitaristas-. Es verdad que entonces se haría patente que se trata de una postura controvertida que algunas doctrinas comprehensivas no estarán dispuestas a aceptar. Pero ello no debería suponer ningún problema serio, mientras se pueda argumentar que es la postura correcta o la más correcta ${ }^{25}$.
I J. Rawls, El liberalismo político, Barcelona, Crítica, 1995, p. 95.

2 Véase Brian Barry, «John Rawls and the Search for Stability», Ethics, núm. 105, 1995, pp. 886 ss.

${ }^{3}$ He aquí un ejemplo sacado de El liberalismo político, p. 93: «... la idea de razonabilidad[...] es parte de un ideal político de ciudadanía democrática que incluye la idea de razón pública. El contenido de este ideal incorpora también lo que los ciudadanos libres $\mathrm{e}$ iguales pueden exigirse mutuamente en lo que respecta a sus respectivas doctrinas comprehensivas. En este caso, no pueden exigir nada que sea contrario a lo que las partes, en tanto que representantes suyos, podrían conceder en la posición original».

${ }^{4} \mathrm{La}$ acusación de que su noción general de «razonable» es circular $y$, por tanto, poco informativa 0 relevante la ha oído Rawls repetidamente. Para un ejemplo de ello, y para el caso concreto de las doctrinas comprehensivas, véase Chantal Mouffe, The Democratic Paradox, Londres, Verso, 2000, p. 26.

5 Véase B. Barry, «How not to Defend Liberal Institutions», en R. B. Douglass, G. Mare y H. Richardson, Liberalism and the Good, Nueva York y Londres, Routledge, 1990, pp. 44-58.

${ }^{6}$ Pensemos, por ejemplo, en lo que defiende la doctrina católica sobre la homosexualidad, la anticoncepción, etc.

7 «How not to Defend Liberal Institutions», p. 53.

8 «John Rawls and the Search for Stability», p. 901.
9 Liberalismo político, pp. 53 y ss.

10 Dice Rawls: «En un caso particular, alguien puede, evidentemente, sostener una doctrina razonable de un modo irrazonable, por ejemplo, con ofuscación o por capricho. Y ello no conlleva que la doctrina sea en sí irrazonable. Una doctrina razonable es una doctrina que puede ser defendida de un modo razonable», El liberalismo político, p. $91 \mathrm{n}$.

"Véase ibid., p. 94.

12 L. Wenar y S. Scheffler coinciden más o menos con Barry en destacar los aspectos más controvertidos -inaceptables para muchas doctrinas comprehensivas- del liberalismo político. a) Wenar, por ejemplo, carga contra la noción de consenso por superposición y tres de los conceptos relacionados con ella. Para poder explicar por qué es necesario el consenso por superposición, Rawls tiene que recurrir a la idea de cargas del juicio; para poder explicar si será posible, tiene que recurrir a una psicología moral particular; para poder explicar cómo se mantendrá, tiene que recurrir al constructivismo político. Pues bien, según Wenar, estos conceptos difícilmente serán aceptados nunca por todas las doctrinas comprehensivas $\mathrm{y}$, por lo tanto, difícilmente se generará jamás un consenso por superposición. Véase $\mathbf{L}$. Wenar, «Political Liberalism: An Internal Critique», Ethics, núm. 106, 1995, pp. 32-62. b) Scheffer, por otro lado, sostiene que, paradójicamente, los requisitos que debe satisfacer una concepción de la justicia para poder ser considerada política imposibilitan que se genere un 
consenso por superposición. Es contraproducente cara a lograr tal consenso, requerir que, además de unos principios de justicia, las concepciones de la justicia sostengan determinadas creencias -como la tesis de las cargas del juicio- sobre su estatus epistemológico. Véase S. Scheffler, «The Appeal of Political Liberalism», Ethics, núm. 105, 1994, p. 19.

13 Véase W. Kymlicka, «Two Models of Pluralism and Tolerance», en D. Heyd (ed.), Toleration: An Elusive Virtue, Princeton, Princeton University Press, 1996, pp. 81-103.

${ }^{14}$ En este sentido, Kymlicka estaría de acuerdo con Rawls que la noción de «razonable» no se basa en una perspectiva epistemológica, sino que presupone un punto de vista moral o ético.

is «Two models of Pluralism and Tolerance», pp. 91-92.

16 Ibid., pp. 83 ss.

17 O constituía una suposición correcta cuando Kymlicka publicó su texto (en el año 1996), pero no lo es ahora, después de la publicación, en el año 1999, de la segunda versión ampliada de The Law of Peoples. Véase J. Rawls, The Law of Peoples, Cambridge Ma., Harvard University Press, 1999.

${ }^{18}$ Cf. ibid., $\$ 9.3$

19 Ibid., p. 73.

20 «Two Models of Pluralism and Tolerance», p. 889 .

i1 The Law of Peoples, p. 67.

${ }_{22}$ Cf. ibíd., $\$ 7.1$ y 7.2 .
${ }^{23}$ Si sabemos qué aspecto tiene una sociedad justa y no es imposible pensar que las sociedades decentes podrían ser razonables ( $y$, por consiguiente, más justas de lo que son ahora), ¿por qué deberíamos estar dispuestos a aceptar como moralmente correcto o justo un orden social mundial en el que unas sociedades son más justas que otras? ¿Qué podríamos decirles, por ejemplo, a las personas de la sociedad decente que luchan a fin de hacer más justa su sociedad? Posiblemente no podamos lograr en seguida que todas las sociedades sean igual de justas. Tal vez, por razones de prudencia política, ahora mismo sea correcto no plantear la cuestión de qué régimen político es moralmente superior. Sin embargo, esto no tiene nada que ver con la cuestión moral de qué régimen es moralmente superior.

24 Esto y lo que hemos destacado anteriormente nos hacen ver que el interés de la cuestión de la tolerancia, en el fondo, es saber qué hacer con los que no son tolerantes en el sentido que uno quisiera.

${ }_{25}$ Afirma Kymlicka: «en [Political Liberalism] Rawls está menos dispuesto a defender el liberalismo comprehensivo, pero todavía quiere imponer las instituciones políticas liberales. Una respuesta más adecuada, creo, sería continuar defendiendo el liberalismo comprehensivo basado en la autonomía entendida como un valor general, pero ser más cauto a la hora de imponer todo un paquete de instituciones políticas liberales a las minorías (o doctrinas comprehensivas) no liberales» («Two Models of Pluralism and Tolerances», p. 96). 